\title{
Für die Behandlung in nur 1 Sitzung
}

Dentsply Sirona CAD/CAM präsentierte vom 10. bis 12. März Meinungsführern der restaurativen Zahnheilkunde aus aller Welt exklusiv den neuen CEREC Zirconia Workflow in Bensheim. 150 nationale wie internationale CEREC-Erprober und -Trainer erlebten die Arbeitsabläufe mit dem neuen CEREC SpeedFire-Ofen in Vorträgen und Hands-on-Kursen. Roddy MacLeod, Vice President Dental CAD/CAM Systems bei Dentsply Sirona, erläuterte den Teilnehmern den besonders kurzen Verarbeitungsprozess von CEREC Zirconia zu Vollkronen und Brücken in einer einzigen Sitzung. Der bei Zahnärzten aufgrund seiner hohen Biegebruchfestigkeit und Biokompatibilität sehr beliebte Werkstoff, der zudem eine substanzschonende Präparation ermöglicht, lässt sich jetzt zeitsparend trockenfräsen und anschließend im neuen CEREC SpeedFire-Ofen in 10 bis 15 min sintern. In jeweils 1

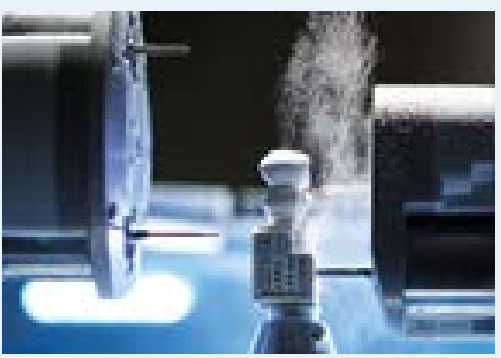
Hands-on-Kurs zur Fertigung einer Einzelkrone und einer Brücke konnten die Zahnärzte den kompletten CEREC Zirconia Workflow durchlaufen. Als großen Vorteil empfanden die Teilnehmer die monolithische Form der Restaurationen, da dadurch kein Risiko für Chipping besteht. Auch die Farbauswahl passt zu den neuen Möglichkeiten: das Material ist ein voreingefärbtes transluzentes Zirkonoxid und in 10 Farben in Anlehnung an den VITA Classic Shade Guide erhältlich.

Nach einer Pressemitteilung der

Dentsply Sirona CAD/CAM, USA - York

www.dentsplysirona.com 